\title{
Osmodehidrocongelación de batata fortificada con Zinc y Calcio
}

\section{Osmodehydrofreezing of the sweet potato fortified with Zinc and Calcium}

\author{
Bosco, Daniela (2,4); Roche, Luis (1,2,3); Della Rocca, Patricia (1,2); Mascheroni, Rodolfo $(1,3)$. \\ (1) Centro de Tecnologías Químicas, CTQ, Universidad Tecnológica Nacional, UTN, Argentina. \\ (2) Departamento de Ingeniería Química, UTN, Facultad Regional Buenos Aires, FRBA, Argentina. \\ (3) Centro de Investigación y Desarrollo en Criotecnología, CIDCA (CONICET y UNLP), Argentina. \\ (4) Escuela de posgrado, UTN, FRBA, Argentina.
}

Contacto: patriciadellarocca@hotmail.com

RECIBIDO: 16/3/2018 - APROBADO: 8/6/2018

\begin{abstract}
Resumen
La batata, Ipomoea batatas L. (Lam), es un alimento muy consumido por sus características nutricionales. Puede considerarse como un alimento funcional por su importante contenido de minerales $(\mathrm{K}, \mathrm{Mg})$ y compuestos con actividad antioxidante (carotenos, vitamina E, antocianinas y polifenoles). El objetivo de este trabajo fue estudiar la osmodehidrocongelación de batatas, que consiste en un proceso previo de deshidratación osmótica/impregnación con Zn y Ca para mejorar sus características nutricionales y texturales y posterior congelación, y para contribuir a evitar algunas carencias nutricionales de la población. Asimismo, se realizó el ajuste de los datos experimentales a modelos de la literatura que describen el comportamiento del proceso de deshidratación osmótica/impregnación (DO/I) y de congelación. El contenido de $\mathrm{Zn}$ y de Ca de las muestras frescas y de las deshidratadas osmóticamente se midió por espectrofotometría de absorción atómica de llama. Se aplicaron satisfactoriamente los modelos de DO de Page (1949), Henderson y Pabis (1961), Midilli et al. (2002), Azuara et al. (1992) y Crank (1975). Posteriormente, se procedió a la congelación en un túnel de bandejas hasta que el centro de los cubos de batata alcanzó una temperatura de $-18{ }^{\circ} \mathrm{C}$. Los tiempos de congelación con y sin pretratamiento de DO se estimaron favorablemente con el modelo de Salvadori-Mascheroni (1991), sin requerir los valores de las propiedades térmicas del alimento congelado ni del cálculo de la entalpía efectiva del cambio de fase.

Palabras clave: Osmodehidrocongelación, batatas, fortificación, Zn, Ca, impregnación, alimentos funcionales.
\end{abstract}

\begin{abstract}
Sweet potato, Ipomoea batatas L. (Lam), is a widely consumed food for its excellent nutritional characteristics. This vegetable can be considered as a functional food due to its higher mineral content $(\mathrm{K}, \mathrm{Mg})$ and antioxidant components as carotenoids, vitamin E, anthocyanins and polyphenols.

In this work, the shelf life of the sweet potatoes was intended to be extended by osmodehydrofreezing. $\mathrm{Zn}$ and $\mathrm{Ca}$ were added during the process in order to increase its level in the final product, avoiding nutritional deficiencies. The adjust of the experimental data was carried out with literature models that describe the bahaviour of the osmotic dehydration/impregnation and freezing process. $\mathrm{Zn}$ and Ca contents in the fresh and OD samples was determined by flame absorption spectrophotometry. Models of the OD like Page, Henderson y Pabis, Midilli et al., Azuara et al. and Crank were applied satisfactorily. Later, the samples were frozen in a tunnel of trays until the center of the sweet potatoes cubes reached $-18^{\circ} \mathrm{C}$. The freezing times of the samples with and without OD pretreatment were estimated favorably with the Salvadori-Mascheroni model without requiring the value of the thermal properties of the frozen food or the calculation of the effective enthalpy of the phase change.

Keywords: Osmodehydrofreezing, sweet potatoes, fortification, $\mathrm{Zn}, \mathrm{Ca}$, impregnation, functional foods.
\end{abstract}

\section{Introducción}

La deshidratación osmótica (DO) es un método de conservación que permite eliminar parcialmente el agua de los tejidos de los alimentos por inmersión en una solución hipertónica, generalmente de azúcares, polialcoholes y/o sales. La fuerza impulsora para el egreso del agua es el gradiente de presión osmótica entre el alimento y la solución. La cinética del proceso es afectada por numerosas variables, entre ellas la temperatura, la concentración de solutos en la solución deshidratante, la relación masa de solución a masa de producto, el grado de agitación, el tiempo de proceso (Kumar Yadav y 
Vir Singh, 2014). Durante la deshidratación osmótica las paredes celulares actúan como membranas semipermeables que permiten ser atravesadas por el agua, principalmente, y, en menor grado, por los solutos que se hallan en la solución. Este último fenómeno se denomina impregnación con solutos. El proceso de deshidratación osmótica/impregnación (DO/I) proporciona productos de humedad intermedia. Debido a que el descenso de la actividad de agua $\left(\mathrm{a}_{\mathrm{w}}\right)$ alcanzado en condiciones prácticas no es suficiente para detener totalmente el deterioro microbiano de este proceso, se requiere la aplicación de otros procesos posteriores para disminuir la actividad acuosa a un nivel seguro. Entre estos procesos se encuentran el secado en sus diferentes formas: convección con aire caliente, microondas, secados combinados, fritura, liofilización, congelación.

Cuando la etapa inicial o pretratamiento de alimentos por deshidratación osmótica/impregnación se combina con una sucesiva congelación, se la denomina osmodehidrocongelación o dehidrocongelación. Las ventajas principales que ofrece este procesamiento respecto de la congelación tradicional sola son:

- Elaboración de productos con características especiales, al poder adicionar durante la deshidratación osmótica/ impregnación minerales y vitaminas con el propósito de fortificar el producto.

- Formación de una capa superficial de soluto (por ejemplo: sacarosa) que impide la salida de compuestos volátiles responsables del aroma del alimento.

- Disminución de la cantidad de agua que se solidificará en el proceso posterior de congelación. Esto conduce a un menor daño de la estructura y a la obtención de una mejor calidad final del producto. Además, se produce un ahorro energético al congelar menor contenido de agua.

- Se facilita el manejo de los productos debido a un menor volumen, ahorro en el uso de embalajes, disminución del espacio requerido en almacenes y vehículos de transporte.

La deshidratación osmótica/ impregnación ha sido estudiada por numerosos investigadores (Azuara, et al., 1992; Lenart y Flink, 1984; Della Rocca, 2010; Silva Paz, 2015; Lovera, 2016).

La creciente demanda actual de productos naturales y nutritivos motiva a desarrollar productos alimenticios fortificados con minerales y vitaminas. Es el caso de la adición de calcio, que presenta las siguientes ventajas:

- Previene la pérdida de masa ósea, vital para prevenir la osteoporosis.

- Interviene en la contracción de los músculos.

- Regula la transmisión nerviosa y del latido cardíaco.

- Regula la función de hormonas y la activación de enzimas intra y extracelulares.

Asimismo, el zinc es otro mineral muy importante en la dieta. Actúa como cofactor en una gran cantidad de reacciones enzimáticas, cumple una función estructural, es regulador de la expresión de genes y es fundamental para fortalecer el sistema inmune (Carbajal Azcona, 2013).

Durante la congelación y posterior descongelación se produce el ablandamiento o pérdida de firmeza de los tejidos vegetales. Estos cambios texturales se relacionan con alteraciones de la pared, laminilla media y membranas celulares de los tejidos vegetales. La impregnación de las frutihortícolas con calcio permite mantener la firmeza del vegetal debido a que los iones calcio tienen la capacidad de interactuar formando puentes con los carboxilos libres de las pectinas. La impregnación en solución de calcio combinado con el tratamiento térmico a bajas temperaturas $\left(40-60^{\circ} \mathrm{C}\right)$ constituye un procedimiento global que ejerce una sinergia sobre el efecto reafirmante del tejido vegetal (Lovera, 2016).

También es posible considerar la impregnación con componentes bioactivos como una técnica que se aplica para la obtención de alimentos funcionales.

Durante la congelación disminuye la $\mathrm{a}_{\mathrm{w}}$ en los tejidos del alimento por la cristalización del agua, y de esta forma se reduce el agua líquida del alimento. Tanto la conversión de agua líquida en hielo como la reducción de la temperatura provocan una disminución de las reacciones responsables del deterioro. El inicio de la congelación sucede a una temperatura inferior a la de congelación del agua pura, debido al descenso crioscópico que depende del tipo de solvente y de la concentración (número de partículas) de las sustancias disueltas. La congelación está caracterizada por la nucleación, aparición de los primeros cristales de hielo, y el crecimiento cristalino, etapa posterior que es función de la velocidad de enfriamiento. Cuando las velocidades de congelación son altas se produce gran cantidad de cristales pequeños, intra y extracelulares; por el contrario, a bajas velocidades de enfriamiento se originan cristales extracelulares de mayor tamaño, que crecen a expensas del agua del interior de la célula, que migra hacia el exterior por ósmosis. Una vez definidas las características del alimento (forma, composición, tamaño, empaque) y seleccionado el equipo a utilizar y sus condiciones operativas (temperatura del refrigerante, coeficiente de transferencia calórica, temperatura inicial del producto), interesa principalmente poder calcular el tiempo de residencia del producto en el equipo de congelación. El tiempo de congelación puede calcularse en función de diversos puntos, ya que distintos autores han tomado diferentes temperaturas como representativas del final de la congelación. Como punto de referencia se adoptará el centro térmico, que se define como el punto de mayor temperatura al finalizar la congelación. En alimentos de forma regular (placa plana, cilindro infinito o finito, esfera, prisma) y cuando el fenómeno de transferencia de calor está gobernado por conducción y las condiciones operativas son simétricas, el centro térmico coincide con el centro geométrico de la pieza. Como definición del tiempo de congelación se adopta la presentada por el IIR (1972): “El tiempo efectivo de congelación es el tiempo requerido para bajar la temperatura de su valor inicial a un valor dado en el centro térmico" (Salvadori, 1994).

En el proceso de congelación pueden distinguirse tres etapas:

- Preenfriamiento: El alimento se enfría desde su temperatura inicial (generalmente superior a los $0{ }^{\circ} \mathrm{C}$ ) hasta la temperatura de inicio de cambio de fase.

- Cambio de fase: La matriz alimentaria está integrada por sólidos insolubles y una fase acuosa que contiene una cierta cantidad de sólidos disueltos. Los sólidos disueltos en la solución acuosa de la matriz son los responsables del descenso crioscópico, que es proporcional a su concentración. A medida que desciende la temperatura, el agua de la solución se solidifica y la solución acuosa incrementa su concentración, por consiguiente, se solidifica a temperaturas inferiores al transcurrir el tiempo. A temperaturas muy bajas, inferiores a los $-40^{\circ} \mathrm{C}$, 
todavía existe una fracción de agua sin congelar que es la denominada "agua ligada".

- La temperatura de inicio de congelación es aquella en la que solo aparecen cristales de hielo puro. A medida que transcurre la congelación, empiezan a formarse cristales de agua hasta alcanzar cierta concentración denominada eutéctica. Pueden existir varios puntos eutécticos en un alimento, según su complejidad en la composición.

- Atemperado: Es la etapa final en la que el alimento se enfría hasta la temperatura final de congelación en su centro térmico.

Las curvas de congelación representan la evolución de la temperatura del centro térmico, en función del tiempo. En ella se distinguen las tres etapas anteriores (Gráfico 1).

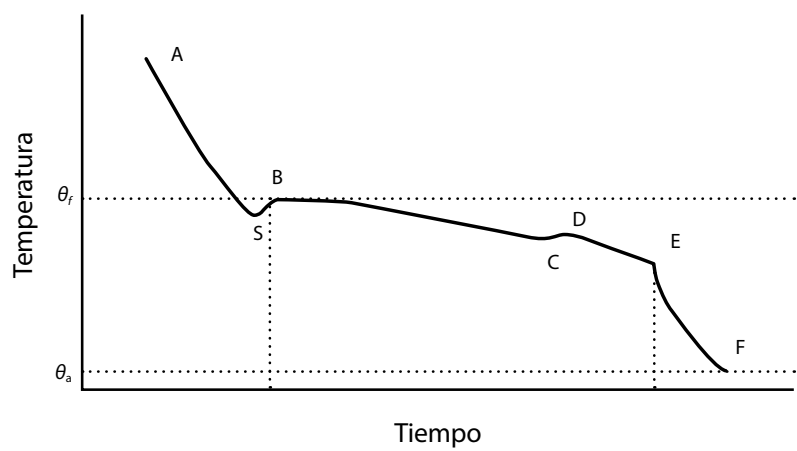

Gráfico 1. Evolución de la temperatura en el centro térmico con el tiempo durante el proceso de congelación AS etapa de preenfriamiento, BE etapa del cambio de fase, EF etapa de atemperado.

El tramo SB corresponde al momento en que el calor liberado durante la congelación de los primeros cristales es superior a la extracción de energía por parte del equipo, por ello se produce en la curva un leve aumento de temperatura desde el subenfriamiento $S$ hasta el comienzo de la etapa de cambio de fase $\mathrm{B}$.

Las curvas de enfriamiento difieren entre una sustancia pura y un alimento (Figura 1). En el caso de una sustancia pura existe una zona de meseta para el cambio de fase. Sin embargo, para un alimento el cambio de fase no se produce a temperatura constante. Se presenta una cierta pendiente, la cual se hace más abrupta a medida que incrementa la velocidad de congelación.

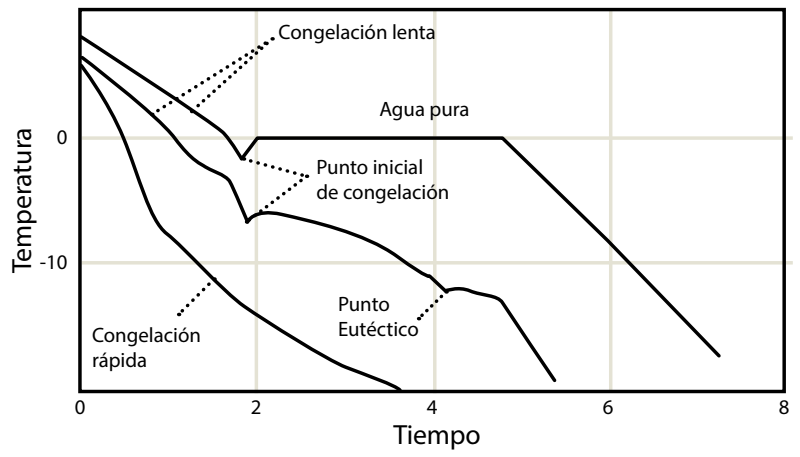

Figura 1. Esquema del proceso de congelación en una sustancia pura (agua) y en un alimento a velocidades de congelación lenta y rápida
El objetivo de este trabajo fue estudiar la osmodehidrocongelación de batatas, que consiste en un proceso previo de deshidratación osmótica/impregnación con $\mathrm{Zn}$ y Ca para mejorar sus características nutricionales y texturales y posterior congelación.

\section{Materiales y Métodos}

La batata o camote (Ipomoea batatas L. o Lam) puede ser considerado un alimento funcional por sus compuestos con actividad antioxidante como carotenos, vitamina E, antocianinas, polifenoles y minerales. La Tabla 1 presenta la composición de la batata.

\begin{tabular}{|l|l|}
\hline Componentes & Cantidad por kg \\
\hline Carbohidratos & $248-344 \mathrm{~g}$ \\
Proteínas & $11,3-18,0 \mathrm{~g}$ \\
Grasas & $3,7-6,0 \mathrm{~g}$ \\
\hline Agua & $640-710 \mathrm{~g}$ \\
Calcio & $280-350 \mathrm{mg}$ \\
Potasio & $2086-2504 \mathrm{mg}$ \\
Fósforo & $420-488 \mathrm{mg}$ \\
Magnesio & $244-315 \mathrm{mg}$ \\
Zinc & $1,12-1,73 \mathrm{mg}$ \\
Hierro & $7-13,8 \mathrm{mg}$ \\
Vitamina A & $8 \mathrm{U} . \mathrm{I}$ \\
Riboflavina & $0,6-0,7 \mathrm{mg}$ \\
Niacina & $6-12,9 \mathrm{mg}$ \\
Ácido ascórbico & $220-400 \mathrm{mg}$ \\
\hline
\end{tabular}

Tabla 1. Composición de la batata (Cusumano, 2013).

\section{Experiencias de deshidratación}

Las batatas fueron lavadas, peladas y luego cortadas en cubos de $1 \mathrm{~cm}$ de arista. Luego se sumergieron en una solución deshidratante: concentración de sacarosa $(40 \% \mathrm{~m} / \mathrm{m})$, cloruro de calcio $(5 \% \mathrm{~m} / \mathrm{m})$, gluconato de $\mathrm{Zn}(0,5 \% \mathrm{~m} / \mathrm{m})$, ácido ascórbico $(5 \% \mathrm{~m} / \mathrm{m})$ y ácido cítrico $(1 \% \mathrm{~m} / \mathrm{m})$. La temperatura de las experiencias fue de $40^{\circ} \mathrm{C}$, la relación masa de solución a masa de batata $(R=4)$ y el nivel de agitación fue de 120-130 rpm.

Durante el proceso de deshidratación osmótica se determinó la variación de peso de la muestra de batata en los intervalos de tiempo: 0, 30, 60, 90, 120, 180, 240 y $300 \mathrm{~min}$. De la misma manera, se registró la variación de humedad a los diferentes tiempos, según método de la AOAC International (1996). Los ensayos se realizaron por triplicado. Asimismo, se determinó la absorción de Ca y Zn por la batata inmersa en la solución deshidratante mediante espectrofotometría de absorción atómica para los tiempos anteriores hasta los $240 \mathrm{~min}$. 


\section{Modelos de deshidratación osmótica}

Las ecuaciones para determinar la humedad $(\mathrm{H})$, los sólidos totales (ST), la pérdida de peso (PP), la pérdida de agua (PA) y la ganancia de sólidos (GS) se detallan a continuación:

$\mathrm{H}(\%)=100-\mathrm{ST}(\%)$

$\mathrm{ST}(\%)=\left(\frac{\mathrm{m}_{\mathrm{s}}}{\mathrm{m}_{\mathrm{o}}}\right) \times 100$

$P P(\%)=\left(\frac{m_{i}-m_{f}}{m_{i}}\right) \times 100$

$\mathrm{PA}(\%)=\left[\left(1-\frac{\mathrm{ST}_{0}}{100}\right)-\left(1-\frac{\mathrm{ST}}{100}\right)\left(1-\frac{\mathrm{PP}}{100}\right)\right] \times 100$

$\mathrm{GS}(\%)=\left[\left(1-\frac{\mathrm{PP}}{100}\right) \frac{\mathrm{ST}^{100}}{100}-\frac{\mathrm{ST}^{0}}{100}\right] \times 100$

$\mathrm{m}_{\mathrm{i}}$, masa inicial de muestra de batata fresca; $\mathrm{m}_{\mathrm{f}}$, masa de muestra deshidratada osmóticamente a tiempo $t ; \mathrm{m}_{\mathrm{s}}$, masa de muestra seca; $\mathrm{m}_{\mathrm{o}}$, masa de muestra antes de secar, $\mathrm{H}(\%)$, porcentaje de humedad; $\mathrm{ST}^{0}$ y ST, contenido de sólidos totales iniciales y en el tiempo, respectivamente.

Se utilizaron los siguientes modelos para ajustar los datos experimentales:

a) Modelo de Page (1949)

b) Modelo de Henderson Pabis (1961)

c) Modelo de Midilli et al. (2002)

d) Modelo de Azuara et al. (1992)

e) Modelo de Crank (1975), que se basa en la segunda Ley de Fick de la difusión.

Los modelos de Page (1949), Henderson y Pabis (1961) y Midilli et al. (2002) plantean una relación directa entre el contenido de humedad y el tiempo de deshidratación.

Modelo de Page (1949)

$\frac{\left(\mathrm{H}_{\mathrm{t}}-\mathrm{H}_{\mathrm{e}}\right)}{\left(\mathrm{H}_{0}-\mathrm{H}_{\mathrm{e}}\right)}=\mathrm{e}^{-\mathrm{K} \mathrm{t}^{\mathrm{N}}}($ parámetros $\mathrm{K} \mathrm{y} \mathrm{N})$

Modelo de Henderson y Pabis (1961)

$\frac{\left(\mathrm{H}_{\mathrm{t}}-\mathrm{H}_{\mathrm{e}}\right)}{\left(\mathrm{H}_{\mathrm{0}}-\mathrm{H}_{\mathrm{e}}\right)}=\mathrm{A} e^{-\mathrm{Kt}}$ (parámetros A y K)

Modelo de Midilli et al. (2002)

$\frac{\left(\mathrm{H}_{\mathrm{t}}-\mathrm{H}_{\mathrm{e}}\right)}{\left(\mathrm{H}_{0}-\mathrm{H}_{\mathrm{e}}\right)}=\mathrm{A} * \mathrm{e}^{-\mathrm{K} \mathrm{t}^{\mathrm{N}}}+\mathrm{B} * \mathrm{t}$ (parámetros $\left.\mathrm{A}, \mathrm{B}, \mathrm{KyN}\right)$

\section{Modelo de Azuara et al. (1992)}

Este modelo se basa en el balance de agua en el alimento. Consiste en una ecuación de dos parámetros que puede predecir la cinética de la deshidratación osmótica y la pérdida de agua en el equilibrio. La principal ventaja es que la pérdida de agua a tiempo infinito se puede estimar utilizando datos obtenidos durante un período relativamente corto de tiempo. Además, su aplicación a los datos experimentales no requiere de las dimensiones, la forma, ni de la estructura del alimento. Sin embargo, como todo modelo empírico, su principal inconveniente es que su validez se limita a las condiciones experimentales para las que se obtuvieron los parámetros. La ecuación que representa el modelo es:

$P A=P A_{\infty}\left[S_{1} t /\left(1+S_{1} t\right)\right]$

Donde $\mathrm{PA}_{\infty}$ es la pérdida de agua en el equilibrio y $\mathrm{S}_{1}$ es la velocidad de pérdida de agua. La ecuación precedente suele linealizarse y escribirse de la siguiente forma:

$\frac{\mathrm{t}}{\mathrm{PA}}=\frac{\mathrm{t}}{\mathrm{PA}_{\infty}}+\frac{1}{\mathrm{~S}_{1} \mathrm{PA}_{\infty}}$

\section{Modelo de Crank (1975)}

Consiste en un grupo de soluciones de la segunda Ley de Fick para diferentes geometrías, condiciones límite y condiciones iniciales. Con este modelo se puede estimar el coeficiente de difusión efectiva del agua $\left(D_{\text {efa }}\right)$ y de los solutos $\left(D_{\text {efs }}\right)$, resolviendo las ecuaciones de manera analítica o numérica. No obstante, las suposiciones que se consideran no siempre son fáciles de lograr, lo que implica grandes limitaciones.

Suposiciones del modelo:

a) El alimento es sólido, homogéneo y uniforme.

b) Se asume que la concentración de los solutos en la solución osmótica se mantiene constante. Esto se logra con una relación masa de solución a masa de alimento muy grande.

c) Por inmersión del alimento en la solución hipertónica se producen dos movimientos difusivos:

- Difusión del agua del interior del alimento hacia la superficie y posteriormente hacia la solución.

- Difusión de solutos desde la solución hipertónica bien agitada hacia el interior del sólido.

d) Las condiciones de equilibrio se determinan experimentalmente y, por lo general, no se alcanza el equilibrio a los tiempos que se consideran en las experiencias.

e) Se considera que no hay efecto de los solutos ganados ni de los solutos perdidos en el proceso de difusión del agua.

f) Se desprecia el encogimiento del alimento debido a la transferencia de masa.

g) Se considera que el único mecanismo para la transferencia de agua es la difusión molecular; si bien este es el principal, también existen otros mecanismos.

h) Se desprecia la resistencia externa a la transferencia de masa lograda con la agitación de la solución hipertónica. Esta suposición no se puede lograr a bajas temperaturas ni a una alta concentración de soluto en la solución.

Por consiguiente, el uso del modelo de Crank (1975) se convierte en un procedimiento empírico que intenta ajustar los datos experimentales para determinadas condiciones de operación y el coeficiente de difusión efectiva del agua resulta un parámetro cinético fuertemente dependiente de las condiciones experimentales y del método matemático.

\section{Determinación de coeficientes de difusión}

La segunda Ley de Fick para la difusión unidireccional en estado transitorio está dada por: 
$\frac{\partial C}{\partial t}=D_{e f} \frac{\partial^{2} C}{\partial x}$

Donde:

C: concentración

t: tiempo

$\mathrm{D}_{\text {ef: }}$ coeficiente de difusión efectivo o aparente

$\mathrm{x}$ : dimensión en la que se produce la difusión, distancia desde el centro de la placa.

Para las siguientes suposiciones y condiciones límite:

$\mathrm{C}=\mathrm{C}_{\mathrm{e}}$ a $\mathrm{t}=0-\mathrm{L}<\mathrm{x}<+\mathrm{L}$

donde $\mathrm{L}$ es el semiespesor de la placa y $\mathrm{C}_{0}$ es la concentración inicial.

$\mathrm{C}=\mathrm{C}_{1}$ a $\mathrm{t}>0 \mathrm{x}= \pm \mathrm{L}$

donde $\mathrm{C}_{1}$ : la concentración en el seno del fluido.

La solución de la ecuación - promediada en el volumen cuando se plantea en tres dimensiones para la difusión del agua en los cubos de batata se detalla a continuación:

$\frac{\left(\mathrm{H}_{\mathrm{t}}-\mathrm{H}_{\mathrm{e}}\right)}{\left(\mathrm{H}_{0}-\mathrm{H}_{\mathrm{e}}\right)}=\sum_{\mathrm{n}=1}^{\infty} \mathrm{C}_{\mathrm{n}}^{3} \exp \left[-\mathrm{D}_{\text {efa }} \mathrm{t}_{\mathrm{n}}^{2}\left(\frac{3}{\mathrm{a}^{2}}\right)\right]$

H se reemplaza por C (concentración) en la Ecuación 12, cuando se calcula la difusividad efectiva de los iones calcio o zinc:

$\mathrm{C}_{\mathrm{n}}=\frac{2 \alpha(1+\alpha)}{1+\alpha+\alpha^{2} \mathrm{q}_{\mathrm{n}}^{2}}$

$\tan q_{n}=-\alpha q_{n}$

$\mathrm{D}_{\text {efa }}$ : coeficiente de difusión efectivo del agua.

Los subíndices 0 , t y e se refieren a las condiciones iniciales, a cualquier tiempo $t \mathrm{y}$ al equilibrio, respectivamente.

a: semiarista del cubo.

$\mathrm{q}_{\mathrm{n}}$ son las raíces positivas no nulas de la Ecuación 14 .

$\alpha$ es la relación entre el volumen de solución y el volumen del producto (batata).

El número de Fourier para la transferencia de agua.

$F_{\text {oa }}=\frac{3 D_{\text {efa }} t}{a^{2}}$

Cuando el número de Fourier para la transferencia de agua es mayor que 0,1 , solamente el primer término de la serie en la Ecuación 12 es significativo y los demás términos pueden despreciarse.

Entonces, las ecuaciones se reducen a las siguientes expresiones cuando se aplica logaritmo natural a ambos miembros:

$\ln \left[\frac{\mathrm{H}_{\mathrm{t}}-\mathrm{H}_{\mathrm{c}}}{\mathrm{H}_{0}-\mathrm{H}_{\mathrm{c}}}\right]=3 \ln \mathrm{C}_{1}-\mathrm{D}_{\text {cra }} \mathrm{q}_{1}^{2} \frac{3}{\mathrm{a}^{2}} \mathrm{t}$

Si se grafican el primer miembro de esta ecuación versus el tiempo de deshidratación empleando los datos experimentales, en ambos casos se obtiene aproximadamente una recta, cuya pendiente y ordenada al origen correspondiente a la ecuación anterior son las siguientes:

Pendiente: - $D_{\text {efa }} q_{1}^{2} \frac{3}{a^{2}}$
Ordenada al origen: $3 \ln C_{1}$

El valor de $D_{\text {efa }}$ puede determinarse a partir de la pendiente de la recta.

Se consideró como criterio de ajuste de los modelos a los datos experimentales, el coeficiente de determinación, $\mathrm{R}^{2}$

$\mathrm{R}^{2}=\frac{\sum_{\mathrm{i}=1}^{\mathrm{N}}\left(\mathrm{V}_{\mathrm{pre}_{\mathrm{i}}}-\overline{\mathrm{V}}\right)^{2}}{\sum_{\mathrm{i}=1}^{\mathrm{N}}\left(\mathrm{V}_{\exp _{\mathrm{i}}}-\overline{\mathrm{V}}\right)^{2}}$

$\overline{\mathrm{V}}=$ promedio de todos los datos experimentales

$\mathrm{N}=$ número de datos experimentales

Subíndices

pre indica valor predicho por el modelo

exp indica valor experimental

\section{Experiencias de congelación}

Las batatas en cubos frescas y las batatas deshidratadas osmóticamente durante $1 \mathrm{~h}$ se congelaron en un túnel con circulación forzada de aire a $-31,5 \pm 1{ }^{\circ} \mathrm{C}$ en bandejas de acero inoxidable perforadas. El coeficiente de transferencia de calor del equipo de congelación se halla en el rango de 20-23 $\mathrm{W} / \mathrm{m}^{2 \circ} \mathrm{C}$. Las temperaturas de las muestras (en su centro) y del aire se registraron con termocuplas de cobre-constantán, conectadas a un adquisidor de datos a intervalos de tiempo de 10 seg. El tiempo de congelación se determinó experimentalmente y se estimó de forma teórica con el modelo de Salvadori-Mascheroni (1991) hasta que el centro térmico, centro de los cubos, alcanzó una temperatura de $-18^{\circ} \mathrm{C}$.

\section{Estimación de tiempos de congelación}

Generalmente, se emplean dos métodos para la predicción de tiempos de congelación: los numéricos y los aproximados. Las soluciones aproximadas, basadas en suposiciones simplificadoras, fueron aplicadas por varios investigadores, entre ellos Cleland et al. (1987) y Salvadori-Mascheroni (1991).

Los métodos numéricos se basan en la siguiente ecuación diferencial en estado no estacionario para un sistema multidimensional:

$\rho c p \frac{\partial T}{\partial t}=\nabla \cdot(k \nabla T)$

Donde $\rho$ es la densidad, cp el calor específico, $\mathrm{k}$ la conductividad térmica, $\mathrm{t}$ el tiempo y $\mathrm{T}$ la temperatura.

En la resolución numérica de la ecuación diferencial se emplean elementos finitos o diferencias finitas. Las diferencias finitas es un método satisfactorio en geometrías regulares, mientras que los elementos finitos es más adecuado cuando se trata de geometrías irregulares.

Salvadori y Mascheroni (1991) desarrollaron un método de predicción de tiempos de congelación de alimentos (Ecuación 21) válido para las formas regulares, cuya fórmula de cálculo es extremadamente simple; solo es necesario conocer dos propiedades termofísicas del producto sin congelar: la difusividad térmica $(\alpha)$ y la conductividad térmica $(\mathrm{k})$ en las 
condiciones de temperatura inicial. El método cuenta con la ventaja de no requerir valores de propiedades térmicas del producto congelado ni del cálculo de la entalpía efectiva del cambio de fase.

La ecuación es válida para un rango amplio de condiciones operativas, simétricas o asimétricas, frecuentes en la congelación industrial. Para la obtención de la ecuación, Salvadori y Mascheroni (1991) emplearon datos experimentales propios y de otros autores, también calculados por métodos numéricos.

Asimismo, el método es válido para una gran variedad de alimentos de composición y estructura diferentes, como carne vacuna entera y picada, distintos pescados, productos frutihortícolas, geles, etcétera. En general, el método se puede aplicar a alimentos con alto contenido inicial de agua.

$\mathrm{t}_{\mathrm{c}, \mathrm{pl}}=\frac{\mathrm{L}^{2}}{\alpha}(\mathrm{a} \mathrm{Tc}+\mathrm{b})\left(\frac{1}{\mathrm{Bi}}+\mathrm{c}\right)(1+\mathrm{Ti})^{\mathrm{n}}(-\mathrm{Ta}-1)^{-\mathrm{d}}$

Donde $t_{c, p l}$ es el tiempo de congelación de una placa plana en $s ; \alpha$, la difusividad térmica del producto sin congelar ( $\alpha=\mathrm{k} / \rho c \mathrm{c})$, en $\mathrm{m}^{2} / \mathrm{s} ; \mathrm{L}$, el semiespesor de la placa en $\mathrm{m} ; \mathrm{T}_{\mathrm{i}}$, la temperatura inicial; $\mathrm{T}_{a}$, la temperatura del refrigerante (aire) y $\mathrm{T}_{\mathrm{c}}$ la temperatura final a alcanzar en el centro térmico (generalmente $-18{ }^{\circ} \mathrm{C}$ ) expresadas todas en ${ }^{\circ} \mathrm{C}$, Bi es el número adimensional de Biot. Las constantes, empíricas, dependen de la geometría. Para placa plana infinita $\mathrm{a}=$ $-1,272 ; b=65,489 ; c=0,184 ; n=0,096$ y $d=1,070$. Las condiciones de aplicación son:

$2{ }^{\circ} \mathrm{C} \leq \mathrm{Ti} \leq 25^{\circ} \mathrm{C},-45^{\circ} \mathrm{C} \leq \mathrm{Ta} \leq-25^{\circ} \mathrm{C}$ y $1 \leq \mathrm{Bi} \leq 50$.

El número de Biot (Bi) es un número adimensional que relaciona la resistencia interna del producto con la resistencia externa a la transferencia de energía y cuya expresión es:

$\mathrm{Bi}=\mathrm{h} \mathrm{L} / \mathrm{k}$

Donde h es el coeficiente de transferencia de calor por convección expresado en $\mathrm{W} /\left(\mathrm{m}^{2 \circ} \mathrm{C}\right)$ y k es la conductividad térmica del producto expresada en $\mathrm{W} /\left(\mathrm{m}^{\circ} \mathrm{C}\right)$.

Para tener en cuenta la influencia de la forma de los alimentos multidimensionales en el tiempo de proceso se han utilizado distintos tipos de factores de forma.

Cleland et al. (1987) han desarrollado varias versiones de estos. La más ampliamente utilizada es la siguiente:

$t_{\text {total }}=\frac{\mathrm{tc}_{\mathrm{pl}}}{\mathrm{E}}$

Donde $t_{c p l}$ es el tiempo de congelación de una placa plana y $E$ es el factor de forma o número equivalente de dimensiones de transferencia de calor. Para una placa infinita $E=1$, para un cilindro infinito $\mathrm{E}=2$ y para una esfera o un cubo $\mathrm{E}=3$.
El tiempo total calculado con la ecuación de Salvadori y Mascheroni (1991) tiene en cuenta los tiempos empleados en cada etapa:

$t_{\text {total }}=t_{p}+t_{f}+t_{a}$

donde $t_{p}$ es el tiempo de preenfriamiento, $t_{f}$ es el tiempo de congelación y $t_{a}$ es el tiempo de atemperado que sería el que transcurre entre el final de la etapa anterior y el tiempo final total del proceso, que en la industria se considera generalmente cuando se alcanza $-18^{\circ} \mathrm{C}$.

Los errores en la estimación de los tiempos de congelación se calcularon mediante la siguiente ecuación:

Error $=($ Valor experimental - Valor teórico $) /($ Valor teórico $)(25)$

\section{Estimación de propiedades físicas y térmicas de la batata}

Se utilizaron las correlaciones de Choi y Okos (1986) para estimar las propiedades de la batata fresca y deshidratada que dependen de la composición del producto y su temperatura.

\section{Resultados}

\section{Estimación de parámetros de los modelos de deshidratación osmótica}

En el Gráfico 2 se presentan los datos experimentales de humedad de la batata en base húmeda (b.h) en función del tiempo de deshidratación osmótica.

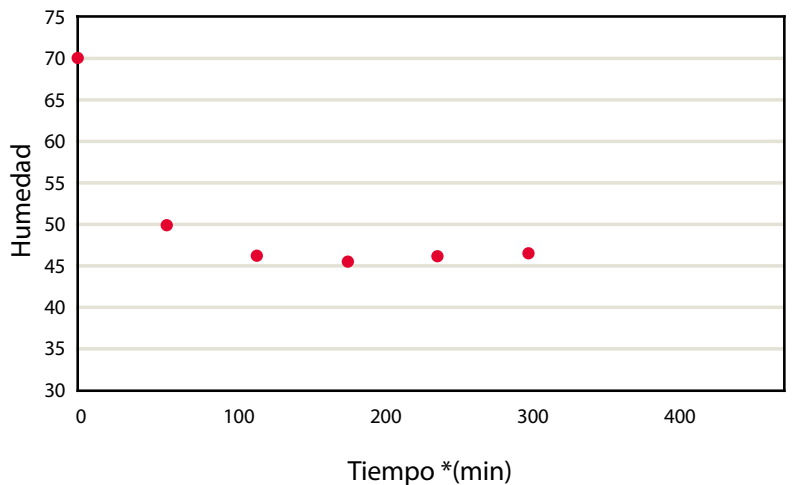

Gráfico 2. Humedad en función del tiempo de deshidratación osmótica para los cubos de batata de $1 \mathrm{~cm}$ de arista para un tiempo de deshidratación osmótica total de $5 \mathrm{~h}$.

\begin{tabular}{|c|c|c|c|c|c|}
\hline Modelo & A & K $\left(\min ^{-1}\right)$ & N & B & $\mathbf{R}^{2}$ \\
\hline Page (1949) & & 0,032 & 0,983 & & 0,9999 \\
Henderson y Pabis (1961) & 0,7457 & 0,023 & & & 0,9196 \\
Midilli et al. (2002) & 0,9988 & 0,0389 & 0,934 & $3,110^{-5}$ & 0,9973 \\
\hline
\end{tabular}

Tabla 2. Parámetros de las ecuaciones de los modelos y bondad del ajuste. 
La humedad tiende a tomar un valor constante a los 120 min del proceso de deshidratación osmótica. Los valores de humedad para tiempos $120 \mathrm{~min}, 180 \mathrm{~min}, 240 \mathrm{~min}$ y $300 \mathrm{~min}$ son muy cercanos entre sí e iguales al valor obtenido al final del proceso de deshidratación osmótica ( $46 \%$ de humedad b.h). Por consiguiente, se puede considerar como humedad de equilibrio este último valor, que fue el que se empleó en los modelos.

En la Tabla 2 se presentan los resultados obtenidos al modelar la transferencia de agua en el proceso de deshidratación osmótica con los modelos de Page (1949), Henderson y Pabis (1961) y Midilli et al. (2002).

El parámetro $\mathrm{N}$ tanto en el modelo de Page (1949) como el de Midilli et al. (2002) se aproximan a 1. Los modelos de Page (1949) y Midilli et al. (2002) son los que ajustaron mejor los datos experimentales. Varios autores encontraron que el modelo de Midilli et al. (2002) describe muy satisfactoriamente la cinética de secado de frutas y vegetales (Onwude, et al., 2016).

Se aplicó el modelo de Azuara et al. (1992) a los datos experimentales de pérdida de agua y se graficó el primer miembro de la ecuación, t/PA, en función del tiempo de deshidratación osmótica, t. En la Tabla 3 se exponen los parámetros correspondientes al modelo. En el Gráfico 3 se presentan los datos experimentales y su correspondiente ajuste con el modelo.

\begin{tabular}{|c|c|c|}
\hline $\mathrm{S}_{1}\left(\mathbf{m i n}^{-1}\right)$ & $\mathbf{P A}_{\infty}$ & $\mathbf{R}^{2}$ \\
\hline 0,04 & 24,8 & 0,9964 \\
\hline
\end{tabular}

Tabla 3. Parámetros del modelo de Azuara et al. (1992).

Este modelo ajustó muy satisfactoriamente los datos experimentales, lo cual puede evidenciarse en el valor de $\mathrm{R}^{2}$ obtenido. Cabe resaltar la ventaja de este modelo que permite predecir la pérdida de agua en el equilibrio sin tener que llegar a él.

A partir de los datos experimentales de humedad inicial (71\%) y humedad final a t: $5 \mathrm{~h}(46 \%)$ se calculó la pérdida de agua en el equilibrio, que resultó ser $25 \%$. Si se compara este valor experimental con el valor obtenido con el modelo de Azuara et al. (1992), 24,8\%, el error diferencial porcentual es del $0,8 \%$ en defecto.

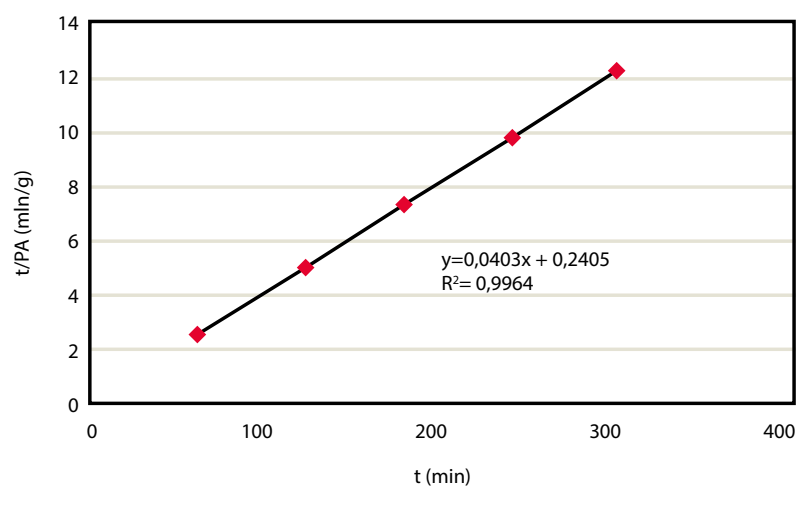

- Datos experimentales

Gráfico 3. Datos experimentales de t/PA en función del tiempo y ajuste con el modelo de Azuara et al. (1992) para la deshidratación osmótica.

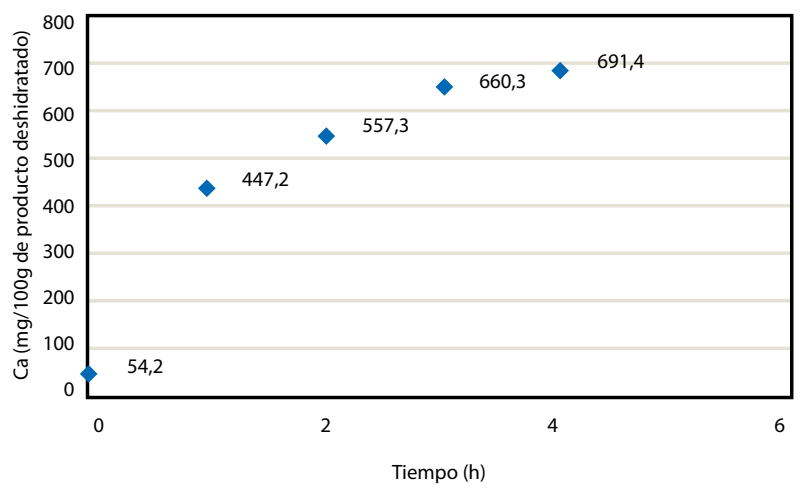

Gráfico 4. Absorción de calcio en batata durante el proceso de deshidratación osmótica.

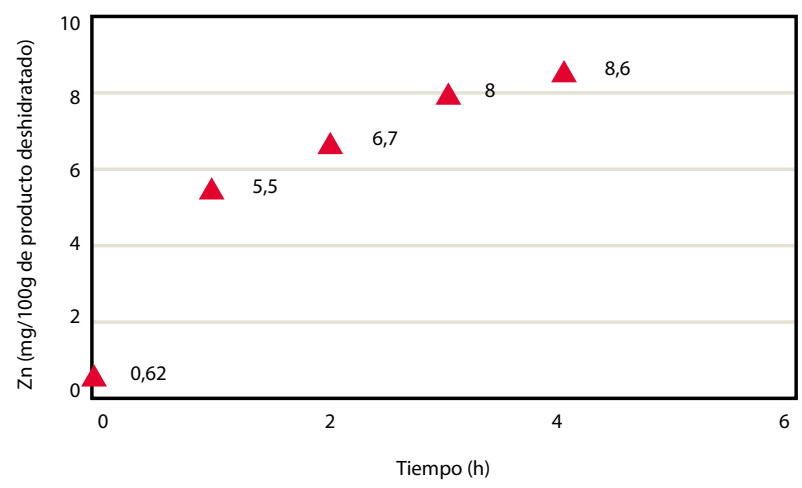

Gráfico 5. Absorción de zinc en batata durante el proceso de deshidratación osmótica.

Los Gráficos 4 y 5 exhiben la absorción de minerales Ca y Zn durante el tiempo de deshidratación osmótica, respectivamente.

La absorción de minerales durante la deshidratación osmótica crece con el tiempo hasta alcanzar un valor máximo aproximadamente a las 4 horas. Asimismo, se puede apreciar que durante la primera hora se absorbe un 63-67\% del valor máximo alcanzado. Esto se cumple tanto para el calcio como para el zinc.

\section{Ajuste de los datos experimentales al modelo de Crank (1975) y cálculo de los coeficientes de difusividad efectiva de los iones calcio y zinc}

En los Gráficos 6 y 7 se presenta el ajuste del modelo de Crank (1975) a los datos experimentales de absorción de calcio y de zinc, respectivamente. En ellos se muestra también la ecuación y la bondad del ajuste, $\mathrm{R}^{2}$.

A partir de la pendiente de las rectas de regresión de los Gráficos 6 y 7 se obtienen los coeficientes de difusión efectiva (Ecuación 16).

El coeficiente de difusión efectiva del ión calcio fue de $2,24 \times 10^{-9} \mathrm{~m}^{2} / \mathrm{s}$ y el del ión zinc fue de $8,7 \times 10^{-10} \mathrm{~m}^{2} / \mathrm{s}$.

Asimismo, se aplicó el modelo de Crank (1975) para determinar el coeficiente de difusión del agua, que resultó 
$\mathrm{Ca}$

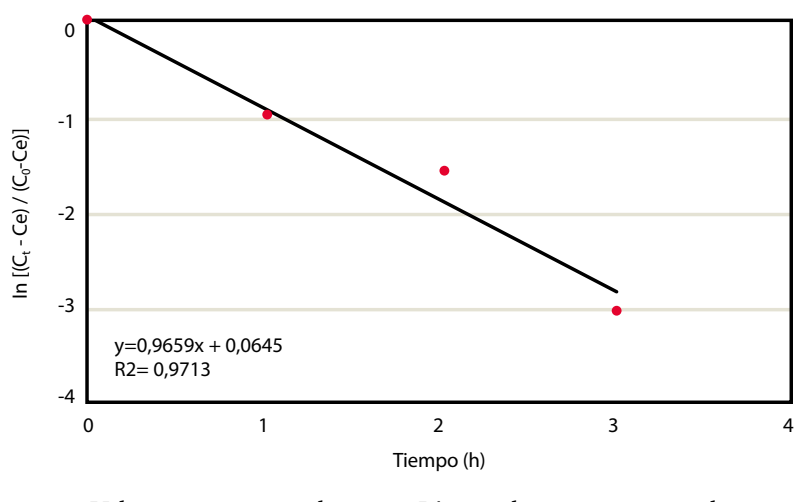

- Valores experimentales - Línea valores experimentales

Gráfico 6. Ajuste de los datos experimentales de absorción de Ca en la batata al modelo de Crank (1975) durante la deshidratación osmótica.

ser $1,4 \times 10^{-9} \mathrm{~m}^{2} / \mathrm{s}$. Este valor se encuentra en el rango de valores de los coeficientes encontrados por Genina Soto et al. (2001) en batata deshidratada osmóticamente en solución de $70^{\circ}$ Brix a $\mathrm{T}=50^{\circ} \mathrm{C}$.

\section{Predicción del tiempo de congelación}

El Gráfico 8 exhibe las curvas de congelación correspondientes a la batata fresca y a la deshidratada osmóticamente. La velocidad de congelación es superior cuando se emplea el pretratamiento de DO (batata deshidratada), tal como puede observarse en las curvas de congelación (temperatura versus tiempo) de pendientes más abruptas en sus distintas zonas y en las que no se aprecian prácticamente zonas amesetadas como en el caso de las sustancias puras.

Los tiempos de congelación de la batata con y sin el pretratamiento de DO se estimaron muy satisfactoriamente aplicando el modelo de Salvadori-Mascheroni (1991) (Ecuaciones 21 y 23). En la Tabla 4 se presentan los valores de tiempos de congelación experimentales y calculados y sus respectivos errores. La batata deshidratada alcanza una temperatura de

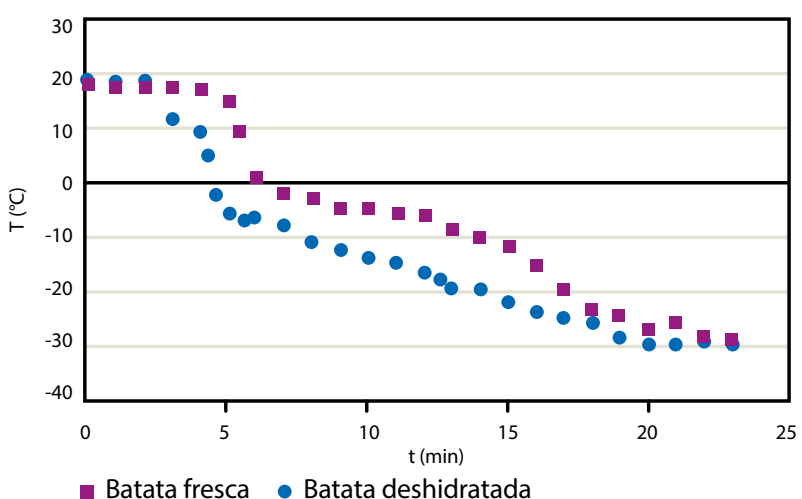

Gráfico 8. Curvas de congelación para la batata fresca y la batata pretratada con deshidratación osmótica (DO) durante $1 \mathrm{~h}$.

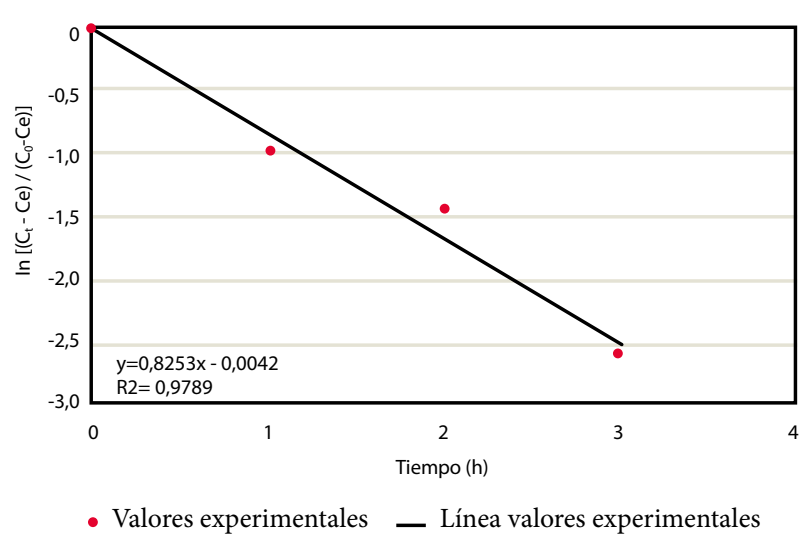

Gráfico 7. Ajuste de los datos experimentales de absorción de Zn en la batata al modelo de Crank (1975) durante la deshidratación osmótica.

congelación final en menor tiempo que la batata fresca debido a que posee menor contenido de agua.

La Ecuación 23 predice el tiempo de congelación con un error del $+1,8$ respecto del experimental para la batata fresca y de $+7,9 \%$ para la batata pretratada por DO.

\begin{tabular}{|l|c|c|c|}
\hline & $\begin{array}{c}\text { Tiempo } \\
\text { experimental } \\
(\mathbf{m i n})\end{array}$ & $\begin{array}{c}\text { Tiempo } \\
\text { calculado } \\
(\mathbf{m i n})\end{array}$ & $\begin{array}{c}\text { Error } \\
(\%)\end{array}$ \\
\hline Batata fresca & 17 & 17,3 & 1,8 \\
\hline Batata con DO 1h & 14 & 15,1 & 7,9 \\
\hline
\end{tabular}

Tabla 4. Tiempos de congelación experimental y calculado con la ecuación de Salvadori-Mascheroni (1991) para batata fresca y batata pretratada por deshidratación osmótica durante $1 \mathrm{~h}$.

\section{Discusión}

- La deshidratación osmótica de cubos de batata resulta ser un proceso eficiente desde el punto de vista energético, ya que permite obtener una reducción significativa de la humedad sin un consumo importante de energía (humedad inicial: 71\%, humedad final: 46\%). La deshidratación osmótica se realiza a temperatura de $40{ }^{\circ} \mathrm{C}$ cercana al ambiente.

- La deshidratación osmótica/impregnación permitió fortificar la batata con calcio y zinc.

- Los modelos de Page (1949), Midilli et al. (2002) y Azuara et al. (1992) ajustaron los datos experimentales muy satisfactoriamente para el proceso de deshidratación osmótica.

- La ecuación de Mascheroni-Salvadori (1991) predijo satisfactoriamente los tiempos de congelación sin requerir los valores de las propiedades térmicas del alimento congelado ni el cálculo de la entalpía efectiva del cambio de fase. Los errores respecto de los tiempos experimentales fueron menores al $10 \%$. 
- El proceso de osmodehidrocongelación disminuye el tiempo necesario de congelación del producto al reducir el contenido de humedad durante el pretratamiento por deshidratación osmótica. De esta forma, se puede lograr un ahorro energético significativo del proceso total.

- Los resultados y las conclusiones alcanzadas aportan información relevante para predecir la tendencia de algunas variables determinantes en la eficiencia del proceso de conservación y pueden contribuir a optimizar las condiciones de proceso a escala industrial.

\section{Referencias}

AOAC International, 1996. Official Methods of Analysis of AOAC International. 16a ed. Gaithersburg: AOAC. Official Method 934.06, first action 1934, revised 1996.

Azuara, E., Beristain, C. I. y García, H.S., 1992. Development of a mathematical model to predict kinetics of osmotic dehydration. En: International Journal of Food Science and Technology, 29(4), pp.239-242.

Carbajal Azcona, A., 2013. Manual de nutrición y dietética [En línea]. Madrid: Universidad Complutense de Madrid. [Consulta: marzo de 2018]. Disponible en: https://www.ucm.es/nutricioncarbajal.

Cusumano, Cosme y Zamudio, Néstor, 2013. Manual técnico para el cultivo de batata (camote o boniato) en la Provincia de Tucumán (Argentina). Tucumán: INTA. ISBN 978-987-679-134-2.

Cleland, D.J., Cleland, A.C. y Earle, R.L., 1987. Prediction of freezing and thawing times for multidimensional shapes by simple methods. Part I: Regular shapes. En: Int. J. Refrig., 10, pp.156.

Crank, J., 1975. The mathematics of diffusion. 2a ed. London: Clarendon Press.

Choi, Y. y Okos, M.R., 1986. Effects of temperature and composition on the thermal properties of foods. En: Le Maguer, M. y Jelen, P., ed. Food engineering and process application. Vol.1. Londres: Elsevier. pp.93-101.

Della Rocca, P., 2010. Secado de alimentos por métodos combinados: deshidratación osmótica y secado por microondas y aire caliente. Buenos Aires: UTN FRBA. (Tesis de maestría).
Genina-Soto P., Barrera-Cortés J., Gutiérrez-López G.F. y Azuara, N.E., 2001 Temperature and concentration effects of osmotic media on OD profiles of sweet potato cubes. En: Drying Technol., 19, pp.547-558.

Henderson, S.M. y Pabis, S., 1961. Grain drying theory. En: I. Agric. Eng. Res., 6(3), pp.169- 174.

IIR, 1972. Recommendations for the processing and handling of frozen foods. 2a ed. Paris: International Institute of Refrigeration.

Kumar Yadav, A. y Vir Singh, S., 2014. Osmotic dehydration of fruits and vegetables: a review. En: Journal of Food Science and Technology, 51(9), pp.1654-1673.

Lenart, A. y Flink J., 1984, Osmotic concentration of potato. I. Criteria for the end-point of the osmosis process. En: Journal Food Technology, 19, pp.45-63.

Lovera, N.N., 2016. Estudio de la impregnación con calcio en la preservación de papaya. La Plata: UNLP. (Tesis de doctorado).

Mascheroni, R.H., 1982. The utilization of numerical methods for solution of the heat balance during the thawing of meat blocks under industrial conditions. En: Lat Am J Heat Mass Transf., 6, pp.13.

Midilli, A., Kucuk, H. y Yapar, Z., 2002. A new model for single layer drying. En: Dry Technol., 20(7), pp.1503-1513.

Onwude, D., Hashim, N., Janius, R., Nawi, L. y Abdan, K., 2016. Modeling the thin layer drying of fruits and vegetables: a review. En: Comprehensive Reviews in Food Science and Food Safety, 15, pp.599-618.

Page, G.E., 1949. Factors influencing the maximun rates of air drying shelled corn in thin layers. Purdue: Department of Mechanical Engineeering. (Tesis de Maestría).

Salvadori, V.O. y Mascheroni R.H., 1991. Prediction of freezing and thawing times of foods by means of a simplified analytical method. En: Journal of Food Engineering, 13(1), pp.67-78.

Salvadori, V.O., 1994. Transferencia de calor durante la congelación, el almacenamiento y la descongelación de alimentos. La Plata: UNLP. (Tesis de Doctorado).

Silva Paz, R. J., 2015. Osmodehidrocongelación de papa (Solanum Tuberosum) impregnada con vitamina C y calcio envasada en atmósfera modificada. Entre Ríos: UNER. (Tesis de Doctorado). 